\title{
Resource Management with Limited Capability of Fixed Relay Station in Multi-hop Cellular Networks
}

\author{
Jemin Lee and Daesik Hong \\ Yonsei University \\ Republic of Korea
}

\section{Introduction}

The purpose of this chapter is to develop a resource management technique to utilize resource efficiently in multi-hop cellular networks. Multi-hop cellular networks have been proposed as a way to enhance throughput and extend coverage (Cho \& Haas, 2004). This enhancement can in general be achieved by deploying relay stations in conventional cellular networks. The advantage of multi-hop networks arises from the reduction in the overall path loss achieved by using a relay station between a base station and a mobile station. Moreover, deeply shadowed mobile stations can be supported by using relay stations to bypass obstacles.

Even though the multi-hop transmission has advantages, it also carries a penalty: the need for additional resources to transmit data in multi-hop manner (for example, in two-hop transmission, two time slots or frequency channels for the base station-relay station link and the relay station-mobile station link) (Lee et al., 2008). Hence, this penalty of multi-hop transmission is represented by 'worms', which devour resources (Ju et al., 2009).

The trade-offs associated with the multi-hop networks make it difficult to assess their overall performance. For mobile stations with quality-of-service (QoS) requirements guaranteed by single-hop transmission with few resources, multi-hop transmission could end up wasting additional resources through multiple hops, even though it may provide higher end-to-end data rates. In other words, a higher end-to-end data rate does not guarantee higher efficiency in resource utilization. Hence, the amount of resources required to guarantee QoS should be considered when assessing multi-hop transmission performance and the performance can be different depending on a mobile station.

In addition, the infrastructure cost increases almost linearly with the equipment capability (Johansson et al., 2004), and it is impossible to change the capability of the installed equipment flexibly according to the change of the required capability. Hence, a relay station has the limited and determined capability. Due to the limited capability, some of mobile stations cannot transmit in multi-hop if all capability of relay station has been already fully used for the other mobile stations. Hence, the resource management for assigning the 
limited capability to the mobile station, who can take more advantage of multi-hop transmission, is required.

In this chapter, for utilizing the resources efficiently, the resource management considering both the different multi-hop gains of each mobile stations and the limited capability of relay station is provided. First of all, a brief explanation about multi-hop cellular networks is provided in Section 2. Then, the transmission mode selection is discussed as a way to determine whether multi-hop or single-hop transmission is the transmission mode most appropriate for minimizing the resources used to guarantee a certain QoS in Section 3. The multi-hop gain is defined as the amount of resources saved by using multi-hop transmission instead of single-hop transmission, and the elements which affect the multi-hop gain are discussed. Based on the affecting elements, two criteria for transmission mode selection are provided and the performance of them is also verified.

In Section 4, the multi-hop user admission is discussed as a way to determine which mobile station should be admitted or rejected to transmit data in multi-hop for maximizing the achievable multi-hop gain using the limited capability of relay station. The multi-hop user admission is formulated as a multi-dimensional knapsack problem, and two efficient heuristic algorithms for multi-hop user admission are introduced and the performance of those algorithms is discussed.

Finally, the structure of resource management including the transmission mode selection and the multi-hop user admission is provided in Section 5.

\section{Multi-hop Cellular Networks}

The network under consideration in this chapter is a downlink orthogonal frequency division multiple access (OFDMA) multi-hop cellular network. The multi-hop system adopted here is the two-hop relaying system, which is known to be the most efficient multihop system with respect to system capacity (Cho \& Haas, 2004). In this system, data can be transmitted from a base station to a mobile station in one of two transmission modes: singlehop transmission or multi-hop transmission via a fixed relay station.

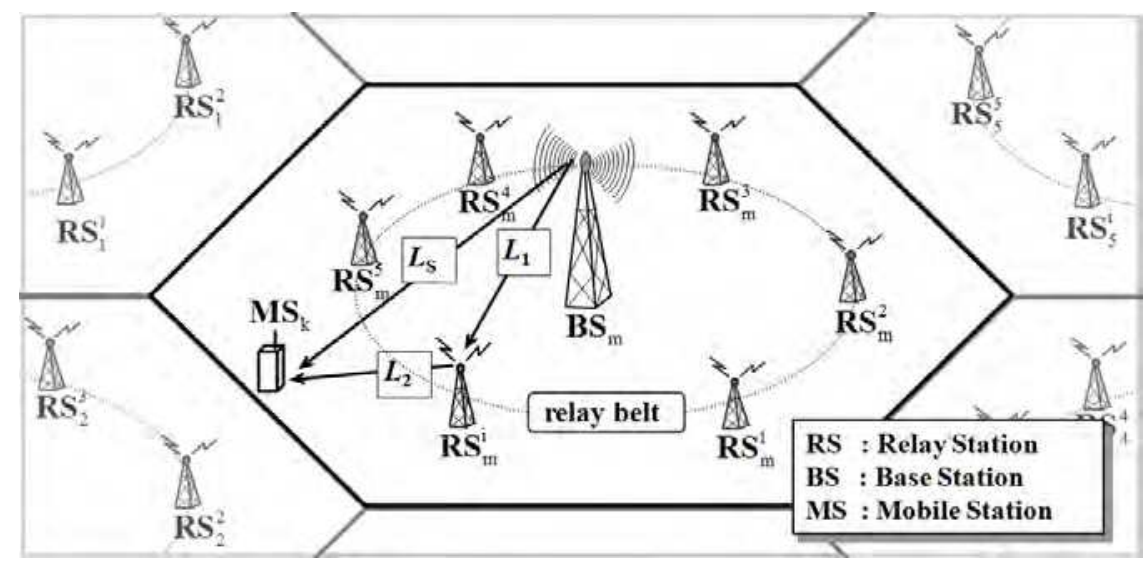

Fig. 1. Downlink multi-hop cellular networks 
Fig. 1 shows an example of the connectivity for this system. In Fig. 1, the $k$ th mobile station, $M S_{k}$, is connected with the $m$ th base station, $B S_{m}$, and $M S_{k}$ uses the $i$ th fixed relay station in the $m$ th cell, $R S_{m}^{i}$, for multi-hop transmission. A number of fixed relay stations are placed on the relay belt. All the fixed relay stations are regenerative relays, so they decode data received from the base station and then forward it to the target mobile stations. In this system, three kinds of links are formed. The base station-fixed relay station links and the fixed relay station-mobile station links occurring with two-hop transmission are denoted by the link for the first hop $\left(L_{1}\right)$ and the link for the second hop $\left(L_{2}\right)$, respectively. The link for single-hop transmission $\left(L_{S}\right)$ also denotes the base station-mobile station link. Generally, it is assumed that $L_{1}$ has good channel condition for a line-of-sight (LOS) environment, and $L_{2}$ and $L_{S}$ are in a non line-of-sight (NLOS) environment (Liu et al., 2006). The LOS assumption can be satisfied by deploying fixed relay stations at selected locations, such as on top of a blinding.

As QoS parameters which can be handled in the physical layer, the target data rate and the target bit-error-rate (BER) can be considered. In the regenerative relay, errors generated at each hop are propagated to the next hop. The sum of the target bit-error-rates for each hop in two-hop transmission should therefore be equal to or less than the target bit-error-rate in two-hop transmission, $B^{T}$, as $\sum_{i=1}^{2} B_{L_{i}}^{T} \leq B^{T}$ where $B_{L_{i}}^{T}$ is the target bit-error-rate on the link of the $i$ th hop (Boyer et al., 2004).

In addition, the end-to-end data rate from a base station to a mobile station is determined by the minimum data rate among the rates in each hop (Jing et al., 2005). Hence, the target data rate for each hop should be equal to or greater than the target data rate, $R^{T}$, as $R_{L_{i}}^{T} \geq R^{T}$ where $R_{L_{i}}^{T}$ is the target data rate on the link of the $i$ th hop.

In OFDMA systems, each subcarrier can obtain a different channel gain. So, the received signal to interference and noise ratio (SINR) on the $n$th subcarrier of the link $L$ $\left(L \in\left\{L_{S}, L_{1}, L_{2}\right\}\right)$ can be defined as

$$
\Gamma_{L}(n)=\frac{G_{L}(n) \cdot P_{I}}{\sum_{j \neq m} \alpha_{j}(n) \cdot G_{I_{j}}(n) \cdot P_{I}+\eta}, \quad \forall L,
$$

where $\eta$ is the additive Gaussian noise power and $G_{L}(n)$ is the channel gain of link $L$ on the $n$th subcarrier. $I_{j}$ is the link between an interferer in the $j$ th cell and the target node, and $P_{I}$ is the transmission power of the transmitter on that link.

If it is assumed that every link in a cell utilizes different resources and resources used for transmission on a link are also reused at the same link in other cells concurrently, then the link $B S_{m}-R S_{m}^{i}$ and the link $B S_{j}-R S_{j}^{i}(j \neq m)$ use the same resources. Hence, in single-hop transmission, $L$ is the link $B S_{m}-M S_{k}, I_{j}$ is the link $B S_{j}-M S_{k}$, and $P_{I}=P_{B S}$, where $P_{B S}$ is the transmission power of the base station in (1). In addition, in two-hop transmission, $L$ is the link $B S_{m}-R S_{m}^{i}, I_{j}$ is the link $B S_{j}-R S_{m}^{i}$, and $P_{I}=P_{B S}$ on the first hop. $L$ is the link $R S_{m}^{i}$ - 
$M S_{k}, I_{j}$ is the link $R S_{j}^{i}-M S_{k}$, and $P_{I}=P_{F R S}$ on the second hop where $P_{F R S}$ is the transmission power of the fixed relay station.

Some of subcarriers are not being used when the mobile stations do not require all of subcarriers in OFDMA systems. Hence, $\alpha_{j}(n)$ in (1) is adopted to express the loading state of the $n$ the subcarrier in the $j$ th cell. $\alpha_{j}(n)=1$ if the $n$th subcarrier is used to transfer data in the $j$ th cell, while $\alpha_{j}(n)=0$ otherwise. Therefore, the average loading state of the $j$ th cell, $\rho_{j}$, is defined as

$$
\rho_{j}=\frac{1}{N} \sum_{n=1}^{N} \alpha_{j}(n)
$$

where $N$ is the total number of subcarriers in a cell. In addition, $\rho_{j}$ becomes one when there is full loading.

With adaptive modulation coding (AMC), the throughput on the link $L$ is expressed as the function of $\Gamma_{L}(n)$ and $B_{L}^{T}$, as follows:

$$
\begin{aligned}
R_{L}(n) & =f\left(B_{L}^{T}, \Gamma_{L}(n)\right) \\
& =\frac{1}{T_{s}} \log _{2}\left(1+\frac{-1.5}{\ln \left(5 \cdot B_{L}^{T}\right)} \cdot \Gamma_{L}(n)\right), \forall L, n,
\end{aligned}
$$

where $T_{s}$ is the symbol duration (Qiu and Chawla, 1999). The number of subcarriers on link $L$ required to guarantee the QoS for $M S_{k}, C_{k, L}$, is determined by $R^{T}$ and $R_{L}(n)$. For instance, when the channel gains of all subcarriers are equal and the average loading state for the other cells is one, then $R_{L}(1)=R_{L}(2)=\ldots=R_{L}$, and $C_{k, L}$ can be defined as

$$
C_{k, L}=\left\lceil R^{T} / R_{L}\right\rceil \forall k, L,
$$

where $\lceil\chi\rceil$ is the least integer equal to or greater than $\chi$. Hence, the total numbers of subcarriers of $M S_{k}$ required to transmit data with a QoS guarantee by single-hop transmission and multi-hop transmission, $C_{k}^{S H}$ and $C_{k}^{M H}$, are respectively defined as

$$
C_{k}^{S H}=C_{k, L_{S}}, C_{k}^{M H}=\sum_{i=1}^{2} C_{k, L_{i}}, \forall k .
$$

A fixed relay station has a limited capability due to the cost, the limitation of power amplifier and so on. In this chapter, the number of supportable subcarriers is considered as the capability of the fixed relay station. It means that the $i$ th fixed relay station can support up to $N_{i, R X}$ subcarriers for receiving on $L_{1}$ (the link between the base station and the relay station) and $N_{i, T X}$ subcarriers for transmitting on $L_{2}$ (the link between the relay station and the mobile station) in a time. Hence, the total number of required subcarriers of each link should be equal or fewer than the supportable number of subcarriers of a fixed relay station in each link as follows:

$$
\sum_{k \in \overline{M U}} C_{k, L_{1}} \leq N_{i, R X}, \sum_{k \in \overline{M U} i} C_{k, L_{2}} \leq N_{i, T X}
$$


where $\overline{M U}_{i}$ is the set of mobile stations who want to use the $i$ th fixed relay station in transmission. Fixed relay stations which have higher capabilities can generally support more subcarriers with higher total power.

\section{Transmission Model Selection: Multi-hop vs. Single-hop}

The multi-hop transmission needs for additional resources, but it achieves more reliable transmission than the single-hop transmission. Due to this trade-off, the multi-hop transmission cannot be better than the single-hop transmission for all users. Hence, the elements, which affect the performance of multi-hop transmission, are investigated and the transmission mode selection for more efficient resource utilization is discussed in this section.

\subsection{Achievable Gain from Multi-hop Transmission}

With respect to efficiency of resources, the gain associated with multi-hop transmission is achieved when subcarriers are saved by transmitting in multi-hop instead of in single-hop. Hence, the multi-hop gain, $S_{k}$, can be defined as the relative ratio of the amount of saved subcarriers to the number of required subcarriers in single-hop transmission as follows (Lee et al., 2008):

$$
S_{k}=\frac{C_{k}^{S H}-C_{k}^{M H}}{C_{k}^{S H}}, \quad \forall k
$$

This approach shows the amount of gain or loss with multi-hop transmission. Thus, if $S_{k}$ has a positive value, that means that the multi-hop transmission is saving subcarriers with guaranteeing the QoS requirements for the $k$ th mobile station. On the other hand, if the value of $S_{k}$ is negative, that implies that the multi-hop transmission is wasting subcarriers.

Fig. 2 presents the multi-hop gain in various environments with the assumption of the equal average loading states of other cells as $\rho_{j}=\rho, \forall j \neq m$ in (2). The distance between the $k$ th mobile station and the base station is denoted by $d_{k}$ and the cell radius is $d_{\text {cell }}$. System parameters in Table 1 are used for simulations. Fig. 2 and the formulas from (1) to (5) show that multi-hop gain is affected by three elements: loading state of other cells, location of the mobile station, and QoS requirements. Due to the long transmission distance in single-hop transmission, $C_{k, L_{S}}$ is generally greater than $C_{k, L_{1}}$ or $C_{k, L_{2}}$. However, for the multi-hop transmission, subcarriers for multi-hops, the sum of $C_{k, L_{1}}$ and $C_{k, L_{2}}$, should be used. By this relation, the multi-hop gain is affected by the loading states of other cells and the location of the mobile station. As the loading state of other cells increases or if the mobile station is located near the cell boundary, the SINRs of the three links decrease. At a lower SINR, the achievable data rate is more sensitive to variation of SINR due to the log function as in (3). This means that the farther the SINR falls, the faster the number of subcarriers required for guaranteeing QoS increases. Hence, in this environment, $C_{k, L_{S}}$ increases more rapidly than the sum of $C_{k, L_{1}}$ and $C_{k, L_{2}}$, so that a bigger multi-hop gain can be achieved. 


\begin{tabular}{|l|l|}
\hline \hline \multicolumn{1}{|c|}{ System Parameters } & \multicolumn{1}{c|}{ Values } \\
\hline \hline System bandwidth & $5 \mathrm{MHz}$ \\
\hline Number of subcarriers & 1024 \\
\hline Path loss exponent (LOS/NLOS) & $2.35 / 3.76$ \\
\hline $\begin{array}{l}\text { Standard deviation of shadowing } \\
\text { (LOS/NLOS) }\end{array}$ & $3.4 \mathrm{~dB} / 8 \mathrm{~dB}$ \\
\hline $\begin{array}{l}\text { Transmission power of base station } \\
\text { /fixed relay station }\end{array}$ & $43 \mathrm{dBm} / 40 \mathrm{dBm}$ \\
\hline Cell radius / Radius of the relay belt & $500 \mathrm{~m} / 250 \mathrm{~m}$ \\
\hline Number of cells & 7 \\
\hline $\begin{array}{l}\text { Number of fixed relay stations } \\
\text { per cell }\end{array}$ & 6, Symmetrically located \\
\hline on the relay belt
\end{tabular}

Table 1. System parameters (for simulations of Fig. 2, Fig. 3, Fig. 5 and Fig. 6)

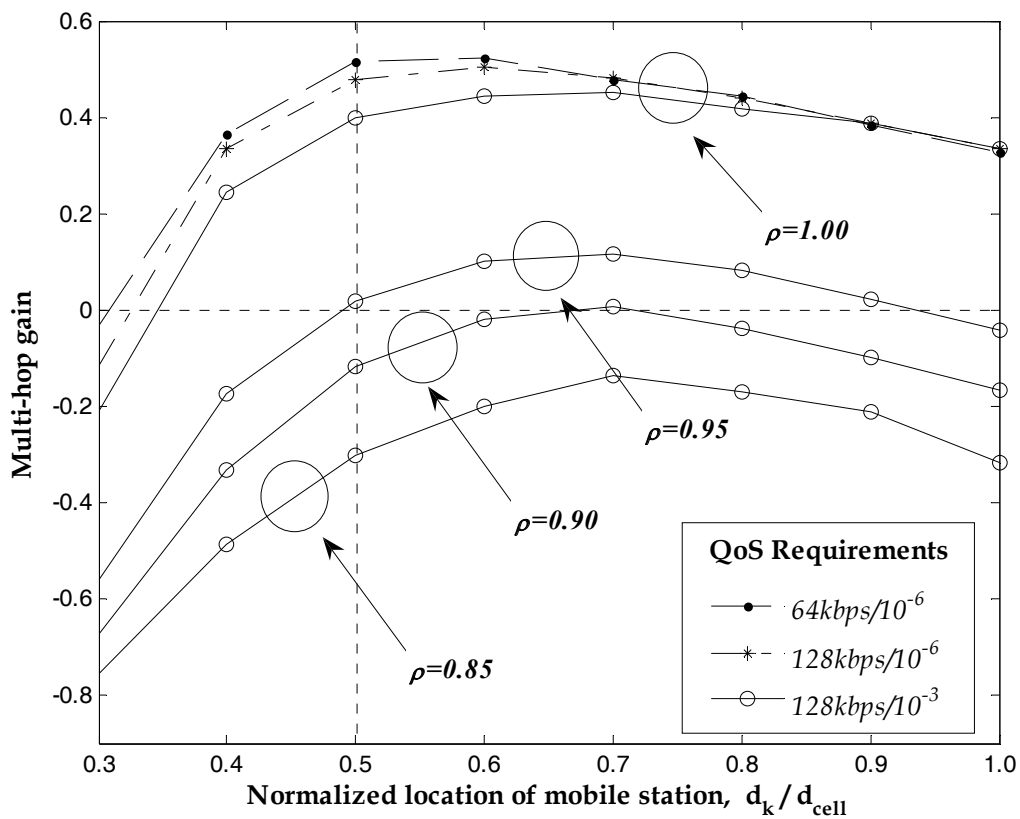

Fig. 2. Multi-hop gain based on QoS requirements (target data rate/target BER), loading state of other cells $(\rho)$ and mobile station location (the mobile station's QoS parameters are $128 \mathrm{kbs} / 10^{-3}$ for all cases except for the case of $\rho=1.0$.) 
However, this increasing multi-hop gain begins to decrease when $d_{k}$ is above a certain value (e.g., $d_{k} / d_{\text {cell }} \geq 0.7$ when $\rho=0.95$ in Fig. 2). The reason for this is that $C_{k, L_{2}}$ is also increasing rapidly due to larger interference as the mobile station moves to the cell edge, causing the difference between $C_{k}^{S H}$ and $C_{k}^{M H}$ to get smaller.

On the other hand, as the mobile station approaches the base station or the loading states of other cells decrease, the SINRs for all three links are increasing. When the SINR has increased enough, the required number of subcarriers becomes small and it is no longer sensitive to the variation in SINR. Hence, in this environment, all of $C_{k, L_{S}}, C_{k, L_{1}}$ and $C_{k, L_{2}}$ are small, so that the multi-hop transmission would waste subcarriers because of the usage of subcarriers for two hops. For this reason, the multi-hop transmission does not have any gain as the loading states of other cells decrease or the mobile station approaches the base station (e.g., $d_{k} / d_{\text {cell }} \leq 0.5$ when $\rho=0.95$ in Fig. 2).

In addition, comparing the upper three lines in Fig. 2 shows that the multi-hop gain has different values depending on the target data rate and the target BER. Even the multi-hop gains for these three cases become similar when the mobile station approaches the cell boundary because of the large amount of interference, a lower target BER induces a higher multi-hop gain because the reliable transmission is more important to mobile stations requiring lower target BERs. The multi-hop gain also varies depending on the target data rate due to the subcarrier allocation process.

In this subsection, the affecting elements on the multi-hop gain, the loading state of other cells, the location of mobile station, and QoS requirement, have been discussed. Since the multi-hop transmission can save (or waste) resources according to those elements, the multihop transmission should be used selectively for efficient utilization of resources. Hence, transmission mode selection is required to determine the appropriate transmission mode for each mobile station: multi-hop transmission or single-hop transmission.

\subsection{Mechanism for Transmission Mode Selection}

The number of required subcarriers changes depending on the transmission mode, the QoS requirements, and the channel condition. To save subcarriers, whichever transmission mode requires fewer subcarriers should be the one selected. Hence, the subcarrier-based criterion, $\Lambda_{k, S}$, is defined as follows (Lee et al., 2008):

$$
\Lambda_{k, S}=C_{k}^{S H}-C_{k}^{M H}, \forall k
$$

If $\Lambda_{k, S}$ is greater than zero, then the selected transmission mode becomes the multi-hop transmission; otherwise, single-hop transmission mode is selected.

In the case where the SINRs for each subcarrier are different, $C_{k}^{S H}$ and $C_{k}^{M H}$ cannot be calculated before the transmission mode is determined because the frequency bands for single-hop and multi-hop transmission may be different. The subcarrier allocation which determines the number of required subcarriers should therefore be performed after determination of the transmission mode. In this case, the average number of required subcarriers $\overline{C_{k}^{\varphi}}$ can be used instead of $C_{k}^{\varphi}$ to calculate $\Lambda_{k, S}$ where $\varphi \in\{S H, M H\} \cdot \overline{C_{k}^{S H}}$ and 
$\overline{C_{k}^{M H}}$ are respectively defined as $\overline{C_{k}^{S H}}=\left\lceil R^{T} / \overline{R_{L_{S}}}\right\rceil$, and $\overline{C_{k}^{M H}}=\sum_{i=1}^{2}\left\lceil R^{T} / \overline{R_{L_{i}}}\right], \forall k$, where $\overline{R_{L}}=(1 / N) \cdot \sum_{n=1}^{N} R_{L}(n)$.

As simpler way to select transmission mode, the distance based criterion can be used. As discussed in Section 3.1, the multi-hop gain changes depending on the locations of mobile stations. Positive gain could be obtained when a mobile station is located near the base station. Hence, the transmission mode can be determined using the following criterion (Lee et al., 2008):

$$
\Lambda_{k, D}=d_{k}-d_{r e f}, \forall k
$$

In (9), $d_{k}$ is $\sqrt{\left(x_{0}-x_{k}\right)^{2}+\left(y_{0}-y_{k}\right)^{2}}$ where $\left(x_{0}, y_{0}\right)$ and $\left(x_{k}, y_{k}\right)$ are the coordinates ( $\mathrm{x}$ and $\left.\mathrm{y}\right)$ of a base station's location and the mobile station's location obtained by a positioning system (e.g., global positioning system), respectively, and $d_{r e f}$ is the reference distance for the transmission mode selection. If $d_{k}$ is greater than $d_{r e f}, \Lambda_{k, D}$ has a positive value and the multi-hop transmission is selected. This means that the multi-hop transmission is applied for mobile stations located outside of the circle with radius $d_{r e f}$.

The performance of the transmission mode selection can be expressed as blocking probability, which is the ratio of the number of unsupportable mobile stations, $K_{U}$, to the total number of mobile stations which access the system, $K_{T}$, or $K_{U} / K_{T}$ as Fig. 3. In this figure, TMS-S and TMS-D represent the transmission mode selections using the subcarrierbased criterion $\left(\Lambda_{k, S}\right)$ and the distance-based criterion $\left(\Lambda_{k, D}\right)$, respectively.

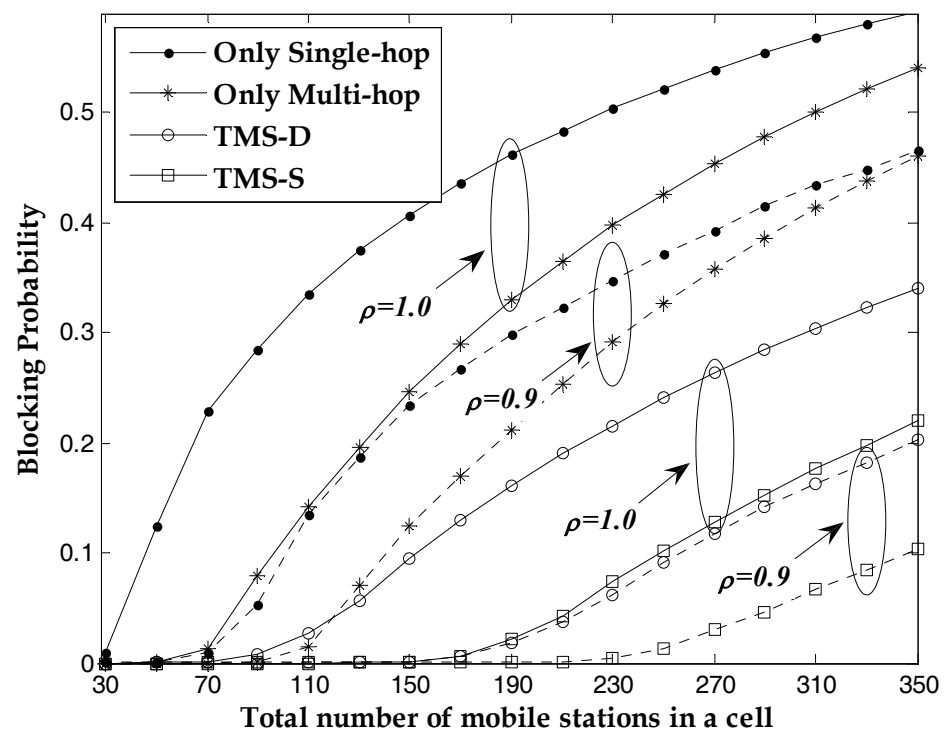

Fig. 3. Comparison of blocking probabilities based on total number of mobile stations in a cell 
In Fig. 3, TMS-D and TMS-S demonstrate better performance, regardless of the loading states of other cells, than the cases where either single-hop transmission or the multi-hop transmission is applied without the selection process. This means that more mobile stations can be supported in a cell with lower blocking probability when the transmission mode selection is applied. Specifically, when the loading states of other cells are 1.0 and the blocking probability is 0.1 , only 45 mobile stations and 95 mobile stations can be supported using conventional single-hop transmission and the multi-hop transmission, respectively. The number of supportable mobile stations increases to 150 with TMS-D and 250 with TMS-S.

\section{Multi-hop User Admission for Relay Stations with Limited Capabilities}

Based on the transmission mode selection in Section 3.2, the multi-hop user and the single-hop user, which denote the mobile stations which select the multi-hop transmission and the single-hop transmission, respectively, are determined. Since all fixed relay stations have the limited capabilities, a fixed relay station may not be able to support all of multi-hop users. When the required subcarriers of multi-hop users are beyond the number of supportable subcarriers in (6), some multi-hop users cannot receive data in multi-hop. Hence, to utilize the limited capability of the fixed relay station efficiently, the multi-hop user admission is required to allow the multi-hop users, which achieve high multi-hop gain, to use a fixed relay station. Therefore, in this section, the problem of multi-hop user admission is discussed and the multi-hop user admission algorithms are presented.

\subsection{Formulation of Multi-hop User Admission}

The multi-hop user admission strategy is formulated to determine the admitted multi-hop users to use the fixed relay station among all multi-hop users for maximizing the total multihop gains which can be obtained from the admitted multi-hop users as follows:

$$
\begin{array}{ll}
\max & \sum_{i=1}^{F} \sum_{k \in \overline{M U}} x_{k} \cdot S_{k} \\
\text { s.t } & \Omega_{1, i}: \sum_{k \in \overline{M U}_{i}} x_{k} \cdot C_{k, L_{1}} \leq N_{i, R X}, \forall i \\
& \Omega_{2, i}: \sum_{k \in \overline{M U}} x_{k} \cdot C_{k, L_{2}} \leq N_{i, T X}, \forall i \\
& \Omega_{3}: x_{k} \in\{0,1\}, \forall k,
\end{array}
$$

where $F$ is the total number of fixed relay stations in a cell, and the $x_{k}$ is the indicator of admission. If $M S_{k}$ is admitted to transmit in multi-hop using the fixed relay station, then $x_{k}=1$, otherwise $x_{k}=0$ and $M S_{k}$ should transmit in single-hop.

For each fixed relay station, there are two constraints from the limited capabilities: $\Omega_{1, i}$ and $\Omega_{2, i}$ based on the $i$ th fixed relay station. The Lagrangian of multi-hop user admission problem, $\operatorname{Lag}\left(u, u^{\prime}\right)$, can be defined as follows:

$$
\begin{aligned}
\operatorname{Lag}\left(u, u^{\prime}\right) & =\sum_{i=1}^{F}\left(\sum_{k \in \overline{M U}_{i}} x_{k} \cdot S_{k}-u_{i} \sum_{k \in \overline{M U}_{i}}\left(x_{k} \cdot C_{k, L_{1}}-N_{i, R X}\right)-u_{i}^{\prime} \sum_{k \in \overline{M U}_{i}}\left(x_{k} \cdot C_{k, L_{2}}-N_{i, T X}\right)\right) \\
& =\sum_{i=1}^{F} \operatorname{Lag}\left(u_{i}, u_{i}^{\prime}\right)
\end{aligned}
$$


where $u_{i}$ and $u_{i}^{\prime}$ are Lagrangian multipliers. Hence, it is shown that the primal problem can be decomposed into sub-problems $Z\left(u_{i}, u_{i}^{\prime}\right)$ where $Z\left(u_{i}, u_{i}^{\prime}\right)=\max \operatorname{Lag}\left(u_{i}, u_{i}^{\prime}\right)$. It means that the parallel multi-hop user admissions for each fixed relay station can work independently since the result of one fixed relay station's multi-hop user admission does not affect the other fixed relay stations' multi-hop user admission. Therefore, we can deal with the multihop user admission problem by decomposing into the independent multi-hop user admission problems for each fixed relay station, which can be formulated as follows:

$$
\begin{array}{ll}
\max & \sum_{k \in \overline{M U}} x_{k} \cdot S_{k} \\
\text { s.t } & \Omega_{1, i}, \Omega_{2, i}, \text { and } \Omega_{3} .
\end{array}
$$

The problem in (12) has the equivalent form with the two-dimensional knapsack problem (TDKP) which is a kind of multi-dimensional knapsack problem (MDKP) (Qiu \& Chawla, 1999). The MDKP is a variant of the classical 0-1 knapsack problem (KP) with more than two knapsacks.

\subsection{Algorithms for Multi-hop User Admission}

As shown in Section 4.1, the multi-hop user admission strategy can be represented as the two-dimensional knapsack problem a kind of MDKP. The KP and the MDKP are proven to be NP-hard, so the optimal solutions of them cannot be obtained in a polynomial time (Akbar et al., 2005). Hence, a heuristic algorithm could be used for multi-hop user admission, and two multi-hop user admission algorithms are presented in this section.

\section{2. 1 Balanced Link Multi-hop User Admission (BL-MUA) Algorithm}

In the multi-hop user admission, the balance between the used resources on $L_{1}$ and those on $L_{2}$ is important. The reason for this is that no additional multi-hop users can be admitted when at least one of $N_{i, R X}$ on $L_{1}$ and $N_{i, T X}$ on $L_{2}$ is fully occupied for other multi-hop users. Hence, the balanced link multi-hop user admission (BL-MUA) algorithm has been proposed in (Lee et al., 2007) considering the balance in admission by adopting the primal effective gradient method (PEGM) (Toyoda, 1975).

The PEGM determines the priority of admission using the new measurement of the aggregate resource. The aggregate resource is to penalize the multi-hop user which requires many subcarriers in the more loaded link. The aggregate resource in multi-hop user admission can be defined as $A_{k}=\left(C_{k, L_{1}} \cdot l_{1}+C_{k, L_{2}} \cdot l_{2}\right) / \sqrt{l_{1}^{2}+l_{2}^{2}}$ where $l_{1}$ and $l_{2}$ are the total numbers of used subcarriers on $L_{1}$ and $L_{2}$ by the currently admitted multi-hop users, respectively. The priority function of the BL-MUA algorithm, $U_{B L, k}$, is set by the multi-hop gain over the aggregate resources, $S_{k} / A_{k}$, as follows:

$$
\mathrm{U}_{B L, k}=\frac{S_{k} \sqrt{l_{1}^{2}+l_{2}^{2}}}{C_{k, L_{1}} \cdot l_{1}+C_{k, L_{2}} \cdot l_{2}} .
$$


If at least one of $N_{i, T X}$ and $N_{i, R X}$ is insufficient for supporting all multi-hop users in $\overline{M U}_{i}$, the multi-hop user with the lowest priority is rejected to use the fixed relay station one by one. The priority function helps to balance between the loading states of two links while rejecting the multi-hop user.

\section{2. 2 Focused Link Multi-hop User Admission (FL-MUA) Algorithm}

The BL-MUA algorithm attaches importance to the balance of used resources in $L_{1}$ and $L_{2}$. However, if fixed relay stations are located in a LOS environment with the base station and the environment of $L_{2}$ is a NLOS (like a general assumption), then the number of required subcarriers to guarantee the same target data rate in $L_{2}$ is much more than that in $L_{1}$, $C_{k, L_{1}}<C_{k, L_{2}}$. This means that more multi-hop users could not be admitted in a fixed relay station generally due to the full loading of $L_{2}$, not that of $L_{1}$ (the supportable subcarriers in $L_{2}$ is exhausted quickly than that in $L_{1}$ ).

Therefore, the multi-hop user admission considering both loading states of $L_{1}$ and $L_{2}$ can be simplified to that considering only that of $L_{2}$. In this case, the multi-hop user admission strategy becomes a simple knapsack problem, not the two-dimensional knapsack problem anymore. Hence, the focused link multi-hop user admission (FL-MUA) algorithm is proposed to focus only on the loading state of $L_{2}$ (Lee et al., 2007). As the priority function in the FL-MUA algorithm, the multi-hop gain per the average number of required subcarriers in $L_{2}$ is used as

$$
U_{F L, k}=S_{k} / C_{k, L_{2}} \text {. }
$$

When the supportable subcarriers of a fixed relay station are not sufficient, the multi-hop users are excluded in a low-priority order one by one.

\section{2. 3 Procedure of multi-hop user admission algorithms}

The process of the multi-hop user admission algorithms progresses independently for each fixed relay station, and the overall procedure is shown by the flow chart in Fig. 4 . If multihop users which want to use the $i$ th fixed relay station exist, the total number of required subcarriers for supporting all multi-hop users in $L_{1}$ and $L_{2}, l_{1}^{o}$ and $l_{2}^{o}$, can be calculated as

$$
l_{1}^{o}=\sum_{k \in \overline{M U}_{i}} C_{k, L_{1}}, \quad l_{2}^{o}=\sum_{k \in \overline{M U} U_{i}} C_{k, L_{2}}
$$

If $l_{1}^{o}>N_{i, R X}$ or $l_{2}^{o}>N_{i, T X}$, then the multi-hop user admission algorithm starts. First of all, the priority values of all multi-hop users are obtained based on the priority function in (11) or (12). After that, the multi-hop user with the lowest priority is selected and the indicator of admission is set to zero. The selected multi-hop user should do single-hop transmission, so the multi-hop user is excluded from the $\overline{M U}_{i}$ and added to the set of single-hop users, $\overline{S U}$. If one or both of the capabilities in the first hop and the second hop, $N_{i, R X}$ and $N_{i, T X}$, are still insufficient after one multi-hop user exclusion, the multi-hop user admission process 
progresses again. At this time, the used resources, $l_{1}$ and $l_{2}$, are changed due to the exclusion of a multi-hop user, so the priority function of the BL-MUA algorithm should be updated. On the other hand, in the FL-MUA algorithm, the updating process is not required and the algorithm just keeps using the previously determined priorities, so it is simpler than the BL-MUA algorithm. The process of the multi-hop user admission continuously works until both of $N_{i, R X}$ and $N_{i, T X}$ are sufficient for supporting all multi-hop users in $\overline{M U}_{i}$.

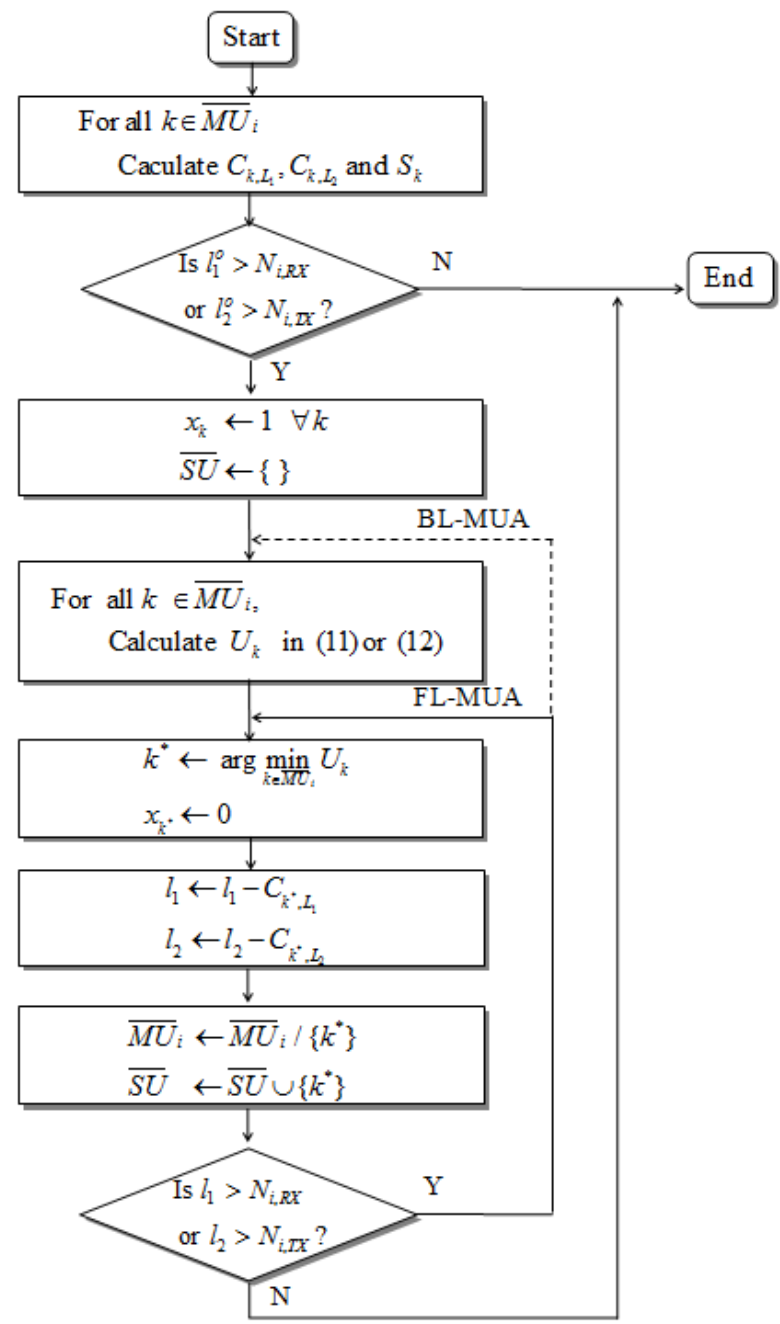

Fig. 4. Flow chart of the multi-hop user admission algorithms (BL-MUA algorithm and FLMUA algorithm) 


\subsection{Performance of Multi-hop User Admission Algorithms}

In this section, the performance of the BL-MUA algorithm and that of the FL-MUA algorithm are evaluated with the assumption that the deployed fixed relay stations have the same capabilities as $N_{R}$ and all capabilities for transmitting and receiving are equal as $N_{i, R X}=N_{i, T X}=N_{R}, \forall i$. The performances of the multi-hop user algorithms are verified with two types of fixed relay stations: the fixed relay station with low capability (L-FRS) and that with high capability (H-FRS). The numbers of supportable subcarriers per fixed relay station are set to 16 for L-FRS and 64 for H-FRS, and the average loading state of other cells is set to one. The other system parameters are the same as Table 1. In addition, the performances of the multi-hop user admission algorithms are compared to the case where multi-hop users are randomly admitted without an admission algorithm (w/o MUA in Fig. 5).

In both multi-hop user admission algorithms, with the higher priority, the supportable subcarriers of a fixed relay station are used for the mobile stations which occupy fewer subcarriers with higher multi-hop gain. Hence, the number of admitted multi-hop users in a fixed relay station can be increased by the algorithms. Those are verified in Fig. 5 .

Fig. 5 presents the number of admitted multi-hop users in a fixed relay station according to two types of fixed relay stations. More multi-hop users can be supported in a fixed relay station by the multi-hop user admission algorithms within the limited capability of fixed relay station compared to the case without multi-hop user admission algorithm.

Moreover, the performance difference between the multi-hop user admission algorithms and the case without the algorithm becomes more significant as the number of multi-hop users increases. When the number of multi-hop users is small, the capability of fixed relay station is generally sufficient for supporting all multi-hop users. Hence, the multi-hop user admission is not actually required and the performance of the multi-hop user admission algorithms is similar to the case without the algorithms. However, the multi-hop user admission becomes meaningful when the fixed relay station cannot support all multi-hop users because of the insufficient capability.

In addition, the performance of BL-MUA algorithm and that of FL-MUA algorithm are similar. The reason for this is from the physical characteristics of $L_{1}$ and $L_{2}$. The number of required subcarriers in $L_{2}$ is much more than that in $L_{1}$ as $C_{k, L_{2}}>C_{k, L_{1}}$. In this case, the priority function of the BL-MUA algorithm in (11) can be approximated to the priority function of the FL-MUA algorithm in (12) as $U_{B L, k}=S_{k} \sqrt{l_{1}^{2}+l_{2}^{2}} /\left(C_{k, L_{1}} \cdot l_{1}+C_{k, L_{2}} \cdot l_{2}\right) \approx S_{k} / C_{k, L_{2}}=U_{F L, k}$. Thus, the similar priority functions are used in both algorithms, so the total numbers of admitted users of them do not have big difference. It implies that the FL-MUA algorithm could obtain similar performance to the BL-MUA algorithm with less complexity.

In addition, in the aspect of total capacity in a cell, more mobile stations can be supported with guaranteeing their QoS requirements regardless of single-hop or multi-hop transmissions using the multi-hop user admission algorithms. This can be verified by Fig. 6 which shows the blocking probability as the number of mobile stations per cell increases. 


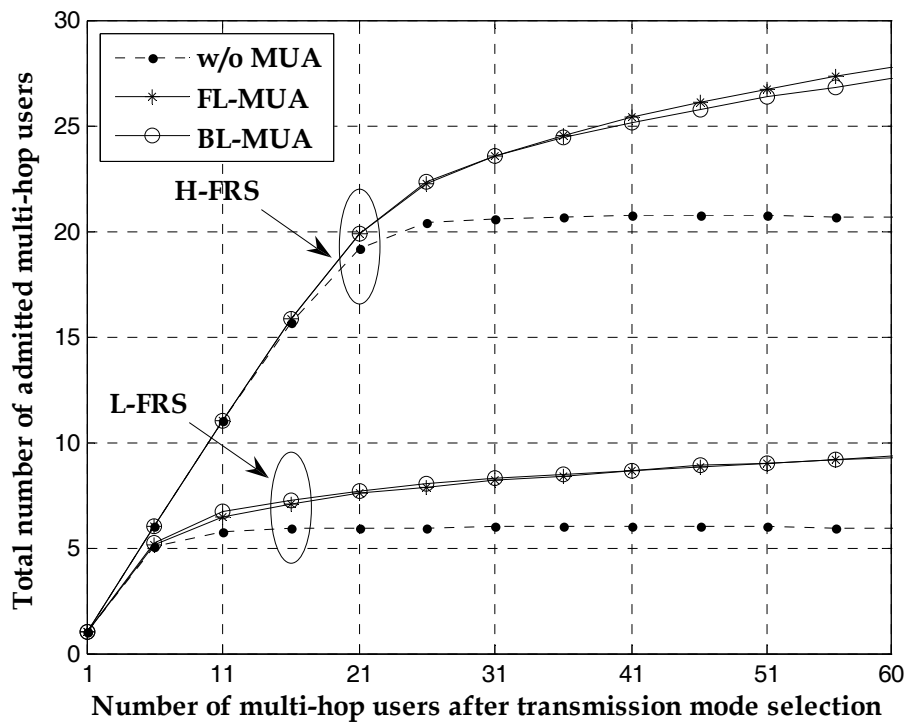

Fig. 5. The total multi-hop gain obtained from the admitted multi-hop users in a fixed relay station according to two types of fixed relay stations

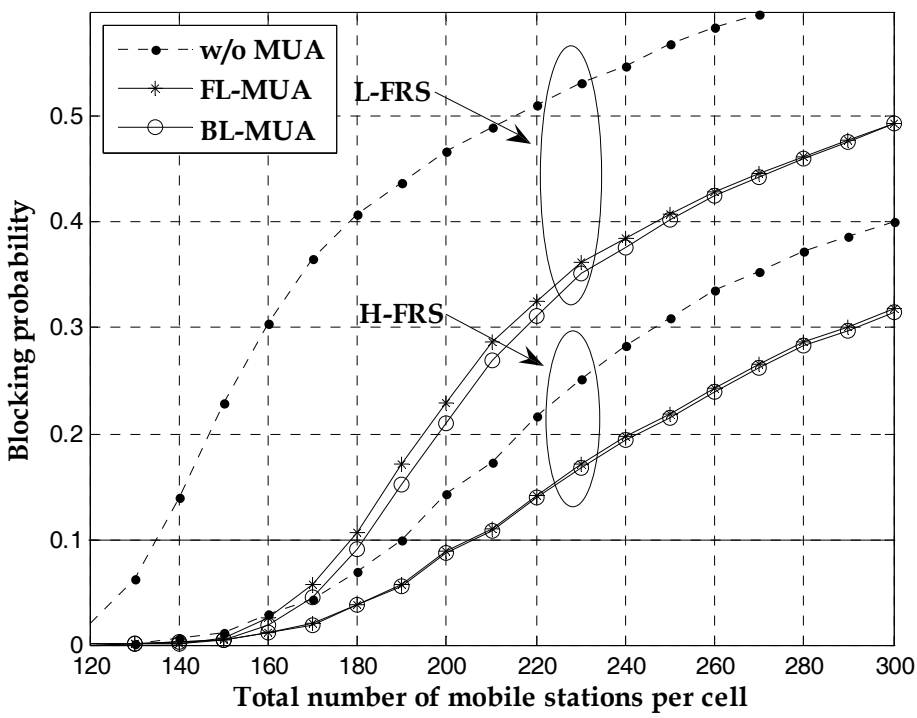

Fig. 6. The blocking probabilities according to two types of fixed relay stations 
The blocking probabilities of the multi-hop user admission algorithms are smaller than that of the case without the algorithm over all range. Specifically, within 0.1 blocking probability, the case without the algorithm can support at most 135 mobile stations with L-FRSs and 185 mobile stations with H-FRSs. On the other hand, the numbers of supportable mobile stations are increased up to 180 mobile stations with L-FRSs and 223 mobile stations with H-FRSs by the multi-hop user admission algorithms. It implies that $33 \%$ and $20 \%$ of the supportable mobile stations in a cell are increased by the multi-hop user admission algorithms with LFRSs and H-FRSs, respectively. Thus, more mobile stations can be supported in a cell with low blocking probability by the multi-hop user admission algorithms.

\section{Overall Structure of Resource Management in Multi-hop Cellular Networks}

In Section 3 and Section 4, the transmission mode selection and the multi-hop user admission have been discussed. The transmission mode determined through the transmission mode selection can be changed after the multi-hop user admission. The reason for this is that some mobile stations should transmit in single-hop due to the limitation of the fixed relay station's capability even though they select the multi-hop transmission in the transmission mode selection. Therefore, the final transmission mode determination and the resource allocation should be performed after the multi-hop user admission. The overall process of the resource management including the transmission mode selection and the multi-hop user admission is summarized as follows:

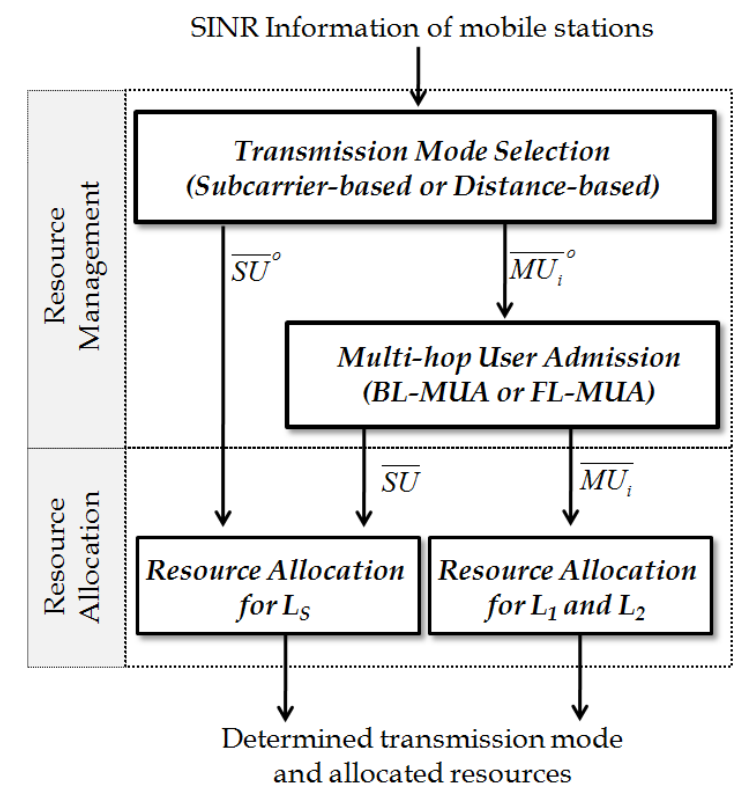

Fig. 7. The overall structure of resource management in multi-hop cellular networks 
1) Once the mobile station set is defined and the required SINR information of the mobile stations are collected in a base station, the resource management process can start. The required SINR information is determined according to the criterion of the transmission mode selection and it could include the received SINRs of $L_{1}, L_{2}$ and $L_{S}$.

2) During the transmission mode selection with a specific criterion (subcarrier-based or distance-based criterion), the set of multi-hop users, $\overline{M U}_{i}^{o}$, and the set of single-hop users, $\overline{S U}^{o}$, are determined.

3) After determining the set of multi-hop users, the multi-hop user admission can be performed. Through a multi-hop user admission algorithm (BL-MUA or FL-MUA algorithm), the mobile stations who can finally receive data in multi-hop, $\overline{M U}_{i}$ and the mobile stations who should receive data in single-hop, $\overline{S U}$, due to the lack of the capability of the fixed relay station are determined.

4) The single-hop users in the sets of $\overline{S U}^{o}$ and $\overline{S U}$ are forwarded to the resource allocation process based on SINR information of $L_{S}$, and the multi-hop users in the set of $\overline{M U}_{i}$ undergoes the resource allocation process based on SINR information of $L_{1}$ and $L_{2}$.

After all of those processes are progressed, the final transmission mode which can maximize the multi-hop gain within the limited capability can be obtained.

According to the transmission mode selection criterion and the multi-hop user admission algorithm, the complexity of this resource management could be changed. In the transmission mode selection, the complexity of the transmission mode selection with the subcarrier-based criterion is $O\left(K_{T} N(\log p) p^{2}\right)$ where $p$ is the required number of digits used in the operations such as square root and multiplication. It is higher than the complexity of transmission mode selection with the distance-based criterion, $O\left(K_{T} p^{2}\right)$ (Lee et al., 2008). Hence, the subcarrier-based selection criterion's complexity is higher than the distance-based selection criterion, but it achieves better performance since it consider more elements for determining an appropriate transmission mode as shown in Section 3.

In the multi-hop user admission, the FL-MUA algorithm is simpler than the BL-MUA algorithm while the BL-MUA algorithm could achieve better performance as shown in Section 4.

Therefore, designers can use appropriate selection criterion and multi-hop user admission algorithm based on what the acceptable complexity level for that system is.

\section{Conclusion}

This chapter provides the resource management for efficient resource utilization in multihop cellular networks. The resource management has two parts: the transmission mode selection and the multi-hop user admission. The transmission mode selection is a way to select an appropriate transmission mode between the multi-hop transmission and the single-hop transmission for saving resources with guaranteeing QoS requirements of mobile stations. Two kinds of selection criteria, subcarrier-based and distance-based criterion, are provided after discussing the elements which affect the multi-hop gains such as the QoS requirements, the mobile station's location, and the loading states of other cells. 
However, due to the limited capability of a relay station, some mobile stations cannot transmit data in multi-hop even though they select the multi-hop transmission mode. Hence, the multi-hop user admission is provided as a way to assign the limited capability of a relay station to the mobile stations which can maximize the multi-hop gain. Since the multi-hop user admission strategy is a NP-hard problem, two heuristic algorithms are provided: the BL-MUA algorithm focused on the load balance between $L_{1}$ and $L_{2}$ and the FL-MUA algorithm focused only on the load state of $L_{2}$. Through the transmission mode selection and the multi-hop user admission, the resources can be used efficiently with supporting more mobile stations with lower blocking probability.

\section{Acknowledgement}

This work was supported by Korea Science and Engineering Foundation through the NRL Program (Grant R0A-2007-000-20043-0).

\section{References}

Akbar, M. M.; Rahman, M. S.; Kaykobaad M. \& Manning, E. G. (2005). Solving the Multidimensional Multiple-choice Knapsack Problem by Constructing Convex Hulls. Computers $\mathcal{E}$ operations research, Vol. 33, No. 5, pp. 1259-1273, ISSN 0305-0548.

Boyer, J.; Falconer, D. D. \& Yanikomeroglu, H. (2004). Multihop Diversity in Wireless Relaying Channels. IEEE Transactions on Communications, Vol. 52, No. 10, pp. 18201830, ISSN 0090-6778.

Cho, J. \& Haas, Z. J. (2004). On the Throughput Enhancement of the Downstream Channel in Cellular Radio Networks through Multihop Relaying. IEEE Journal on Selected Areas in Communications, Vol. 22, No. 7, pp. 1206-1219, ISSN 0733-8716.

Jing, S.; Zhao-yang, Z.; Pei-liang, Q. \& Guan-ding, Y. (2005). Subcarrier and Power Allocation for OFDMA-Based Regenerative Multihop Links. International Conference on Wireless Communications, Networking and Mobile Computing, Vol. 1, pp. 207-210.

Johansson, K.; Furuskar, A.; Karlsson, P. \& and Zander, J. (2004). Relation between Base Station Characteristics and Cost Structure in Cellular Systems. IEEE International Symposium on Personal, Indoor and Mobile Radio Communications.

Ju, H.; Oh, E. \& Hong, D. (2009). Catching Resource-Devouring Worms in Next-Generation Wireless Relay Systems - Two-way Relay and Full-Duplex Relay. IEEE Communications Magazine, Vol. 47, No. 9, pp. 58-65, ISSN 0163-6804.

Lee, J.; Wang, H.; Lim, S. \& Hong, D. (2007). A Multi-hop User Admission Algorithm for Fixed Relay Stations with Limited Capabilities in OFDMA Cellular Networks. IEEE 18th International Symposium on Personal, Indoor and Mobile Radio Communications, pp. 1-5, ISBN 978-1-4244-1144-3, Athens, Sept. 2007.

Lee, J.; Wang, H.; Seo, W. \& Hong, D. (2008). QoS-guaranteed Transmission Mode Selection for Efficient Resource Utilization in Multi-hop Cellular Networks. IEEE Transactions on Wirelss Communications, Vol. 7, Issue 10, pp. 3697-3701, ISSN 1536-1276.

Liu, T. et al. (2006). Radio Resource Allocation in Two-hop Cellular Relaying Network, IEEE Vehicular Technology Conference, Vol. 1, pp. 91-95, ISBN 1-7803-9392-9, May 2006. 
Qiu, X. \& Chawla, K. (1999). On the Performance of Adaptive Modulation in Cellular Systems. IEEE Transactions on Communications, Vol. 47, No. 6, pp. 884-895, ISSN 0090-6778.

Toyoda, Y. (1975). A Simplified Algorithm for Obtaining Approximate Solution to Zero-one Programming Problems. Management Science, Vol. 21, No. 12, pp. 1417-1427, ISSN 0025-1909. 


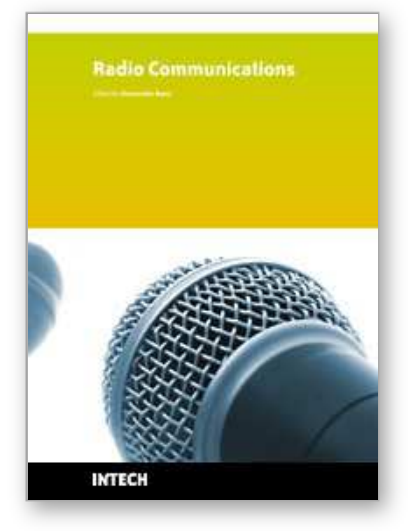

\author{
Radio Communications \\ Edited by Alessandro Bazzi
}

ISBN 978-953-307-091-9

Hard cover, 712 pages

Publisher InTech

Published online 01, April, 2010

Published in print edition April, 2010

In the last decades the restless evolution of information and communication technologies (ICT) brought to a deep transformation of our habits. The growth of the Internet and the advances in hardware and software implementations modified our way to communicate and to share information. In this book, an overview of the major issues faced today by researchers in the field of radio communications is given through 35 high quality chapters written by specialists working in universities and research centers all over the world. Various aspects will be deeply discussed: channel modeling, beamforming, multiple antennas, cooperative networks, opportunistic scheduling, advanced admission control, handover management, systems performance assessment, routing issues in mobility conditions, localization, web security. Advanced techniques for the radio resource management will be discussed both in single and multiple radio technologies; either in infrastructure, mesh or ad hoc networks.

\title{
How to reference
}

In order to correctly reference this scholarly work, feel free to copy and paste the following:

Jemin Lee and Daesik Hong (2010). Resource Management with Limited Capability of Fixed Relay Station in Multi-Hop Cellular Networks, Radio Communications, Alessandro Bazzi (Ed.), ISBN: 978-953-307-091-9, InTech, Available from: http://www.intechopen.com/books/radio-communications/resource-management-withlimited-capability-of-fixed-relay-station-in-multi-hop-cellular-networks

\section{INTECH}

open science | open minds

\author{
InTech Europe \\ University Campus STeP Ri \\ Slavka Krautzeka 83/A \\ 51000 Rijeka, Croatia \\ Phone: +385 (51) 770447 \\ Fax: +385 (51) 686166 \\ www.intechopen.com
}

\author{
InTech China \\ Unit 405, Office Block, Hotel Equatorial Shanghai \\ No.65, Yan An Road (West), Shanghai, 200040, China \\ 中国上海市延安西路65号上海国际贵都大饭店办公楼 405 单元 \\ Phone: +86-21-62489820 \\ Fax: +86-21-62489821
}


(C) 2010 The Author(s). Licensee IntechOpen. This chapter is distributed under the terms of the Creative Commons Attribution-NonCommercialShareAlike-3.0 License, which permits use, distribution and reproduction for non-commercial purposes, provided the original is properly cited and derivative works building on this content are distributed under the same license. 